\title{
Thin Primary Cutaneous Melanomas
}

\section{Associated Detection Patterns, Lesion Characteristics, and Patient Characteristics}

\author{
Jennifer L. Schwartz, M.D. ${ }^{1}$ \\ Timothy S. Wang, M.D. ${ }^{1}$ \\ Ted A. Hamilton, m.s. ${ }^{1}$ \\ Lori Lowe, M.D., ${ }^{1,2}$ \\ Vernon K. Sondak, M.D. ${ }^{3}$
}

Timothy M. Johnson, M.D. ${ }^{1,4,5}$

\begin{abstract}
${ }^{1}$ Department of Dermatology, University of Michigan Medical Center and University of Michigan Comprehensive Cancer Center, Ann Arbor, Michigan.

${ }^{2}$ Department of Pathology, University of Michigan Medical Center and University of Michigan Comprehensive Cancer Center, Ann Arbor, Michigan.

${ }^{3}$ Department of Surgery, University of Michigan Medical Center and University of Michigan Comprehensive Cancer Center, Ann Arbor, Michigan.

${ }^{4}$ Department of Otorhinolaryngology, University of Michigan Medical Center and University of Michigan Comprehensive Cancer Center, Ann Arbor, Michigan.

${ }^{5}$ Department of Surgery, Division of Plastic Surgery, University of Michigan Medical Center and University of Michigan Comprehensive Cancer Center, Ann Arbor, Michigan.
\end{abstract}

Address for reprints: Jennifer L. Schwartz, M.D., Department of Dermatology, University of Michigan, 1910 Taubman Center, Ann Arbor, Ml 481090314; Fax: 734-936-6395; E-mail: jennschw@ umich.edu

Received June 14, 2001; revision received February 4, 2002; accepted April 16, 2002.
BACKGROUND. Public awareness and education may lead to the detection of thinner melanomas, which may result in a decrease in morbidity and mortality rates. Which detection patterns, lesion, and patient characteristics are associated with early detection?

METHODS. Using the University of Michigan prospective melanoma database, the detection patterns, lesion characteristics, and patient characteristics of 1515 consecutive patients with in situ or invasive cutaneous melanomas were reviewed. Tumor thickness (measured in millimeters) was evaluated in relationship to detection patterns (patient, physician, spouse), lesion characteristics (change in color, size, shape/elevation, ulceration, bleeding, tenderness, itching), and patient characteristics (gender, skin type, number of atypical and clinically benign nevi, history of sunburn, personal and family history of melanoma).

RESULTS. Patient characteristics associated with early detection included female gender, at least one atypical nevus, greater than 20 clinically benign nevi, and/or a personal history of melanoma. Skin types I, II, and III, a history of sunburn, and/or a family history of melanoma were also associated with thinner lesions, but these associations were not statistically significant. Lesion characteristics associated with earlier detection included a change in color, size, shape/elevation, and/or itching. Physician-detected melanomas were significantly thinner than patient or spouse-detected lesions.

CONCLUSIONS. Educational campaigns should include increasing melanoma awareness in males and educating the public on the early signs and symptoms. Education should be directed at both high and low-risk groups. Physicians should consider performing total skin examinations routinely on patients. Although they detect a relatively small percentage of all melanomas, physicians detect significantly thinner lesions. Cancer 2002;95:1562-8. (c) 2002 American Cancer Society. DOI 10.1002/cncr.10880

KEYWORDS: melanoma, demographics, detection, signs, symptoms, personal history, family history.

M elanoma is the deadliest form of skin carcinoma. The annual percent increase in the incidence of melanoma exceeds that of any other cancer. ${ }^{1}$ The estimated lifetime risk in the United States for an invasive melanoma is 1 in 75 born in the year $2000 .^{2}$ In 2000, approximately 47,700 invasive melanomas and 28,600 in situ melanomas were diagnosed and approximately 7700 patients died of the disease. $^{3}$

Public awareness and education about melanoma may lead to a true decrease in the incidence of melanoma and have a more immediate impact on the detection of thinner melanomas, ultimately resulting in a decrease in the mortality rate. But who is detecting thin melanomas and where should attention be focused to increase the 
likelihood that a melanoma will be detected earlier? This is the largest prospective study to analyze the relationship between tumor thickness (Breslow depth) and patient demographics, detection patterns, lesion signs and symptoms, and a personal and family history of melanoma. The importance of early detection cannot be overemphasized. The information gained from this study may provide insight on whom to focus educational and screening campaigns by identifying individuals who are more likely to present with deeper lesions.

\section{MATERIALS AND METHODS}

Study approval was granted by the University of Michigan Institutional Review Board for Human Subject Research. Our prospective melanoma database was searched to identify all patients with primary cutaneous invasive and in situ melanomas who were evaluated between January 1, 1998 and December 31, 1999. All histologic slides were reviewed by a University of Michigan melanoma dermatopathologist to confirm the diagnosis of primary cutaneous melanoma. Tumor thickness measured in millimeters $(\mathrm{mm})$ was recorded as the Breslow depth of invasion. Specific clinical data were recorded accurately by a melanoma attending physician and systematically entered prospectively into the melanoma database. Unknown primary and noncutaneous melanomas were excluded. Using these criteria, the study population consisted of 1515 consecutive patients with 1630 lesions.

Patient demographics, detection patterns, lesion characteristics, and personal and family history of melanoma were extracted from the database. 1) Patient demographics included gender, age at diagnosis of first primary melanoma, skin type, number of atypical and clinically benign nevi, and history of sunburn. Skin type categories were defined as follows: I (always burn, never tan), II (always burn, sometimes tan), III (sometimes burn, always tan), IV (never burn, always $\tan ), \mathrm{V}$ (very dark, black skin), and unknown. The number of atypical and clinically benign nevi (based on total body skin examination by a melanoma attending physician and/or histologic diagnosis) was categorized as none, $1-5,6-20$, greater than 20 , or unknown. History of sunburn response categories were yes, no, and unknown. The question was used only to examine tumor thickness in relationship to perceived exposure, not actual or quantitative exposure. 2) Detection patterns determined who first detected the primary melanoma based on patient history. Categories included patient, spouse, physician, friend, sibling, son/daughter, parent, nurse, other, and unknown. 3) Regarding the lesion characteristics of the primary melanoma, patients were questioned
TABLE 1

Patient Demographics ${ }^{\mathrm{a}}$

\begin{tabular}{llll}
\hline & All (\%) & Males (\%) & Females (\%) \\
\hline No. of patients & $1515(100)$ & $774(51)$ & $741(49)$ \\
No. of lesions & $1630(100)$ & $849(52)$ & $781(48)$ \\
Mean depth (mm) & $1.02 \pm 0.08$ & $1.14 \pm 0.11$ & $0.89 \pm 0.11$ \\
Mean age (yrs; first primary) & $52.6 \pm 0.8$ & $56.1 \pm 1.1$ & $48.9 \pm 1.2$ \\
\hline a $\pm 95 \%$ confidence interval. & & & \\
\hline
\end{tabular}

about change in color, size, shape/elevation, ulceration, bleeding, tenderness, and itching. Response categories were yes, no, and unknown. 4) Regarding personal history of melanoma, detection pattern and lesion characteristics were available on each primary melanoma among study patients with multiple primaries. Family history was defined as at least one blood relative diagnosed with melanoma and response categories were yes, no, and unknown.

\section{Statistical Analysis}

Comparisons of continuous variables such as Breslow depth and age were made with Student two-sample $t$ tests, assuming unequal variance. All $P$ values are two tailed. Tests for association of the proportional distribution of categorical variables were made with Pearson's chi-square tests. Descriptive statistics are presented as means $\pm 95 \%$ confidence intervals. The data were analyzed with Microsoft Excel 2000 Software (Microsoft, Bellevue, WA) and SAS Statistical Software (SAS Institute, Cary, NC).

\section{RESULTS \\ Patient Demographics}

The mean Breslow depth of all primaries was $1.02 \mathrm{~mm}$ (Tables 1,2). The majority of lesions were less than 1 $\mathrm{mm}$ thick $(25 \%$ in situ and $43 \%<1 \mathrm{~mm}$ ), whereas only $4 \%$ were greater than or equal to $4 \mathrm{~mm}$ (1-1.99 $\mathrm{mm}$ [18\%], 2-3.99 $\mathrm{mm} \mathrm{[10 \% ]).} \mathrm{The} \mathrm{study} \mathrm{population} \mathrm{con-}$ sisted of about the same number of males and females. The mean Breslow depth of all primaries in females $(0.89 \mathrm{~mm})$ was less than the mean depth in males $(1.14 \mathrm{~mm} ; P<0.005)$. The mean age at diagnosis of the first primary melanoma was 52.6 years. The majority of patients $(72 \%)$ were between the ages of 21 and $65,26 \%$ of the patients were older than 65 years of age, and only $2 \%$ of patients were younger than 21 years of age. Females (48.9 years) were younger than males (56.1 years) at diagnosis of their first primaries $(P<0.001)$.

Most patients had skin types I, II, or III. The mean Breslow depth of lesions in patients with skin types I, 
TABLE 2

Patient Demographics ${ }^{\mathrm{a}}$

\begin{tabular}{|c|c|c|c|c|c|c|}
\hline & \multicolumn{2}{|c|}{ All $(n=1515)$} & \multicolumn{2}{|c|}{ Males $(n=774)$} & \multicolumn{2}{|c|}{ Females $(n=741)$} \\
\hline & №. $(\%)$ & Mean depth (mm) & №. (\%) & Mean depth (mm) & №. (\%) & Mean depth (mm) \\
\hline \multicolumn{7}{|l|}{ Skin type } \\
\hline I, II, III & $1451(96)$ & $1.00 \pm 0.08$ & & & & \\
\hline IV, $\mathrm{V}$ & $39(3)$ & $1.58 \pm 0.71$ & & & & \\
\hline \multicolumn{7}{|c|}{ Atypical nevi } \\
\hline None & $743(49)$ & $1.18 \pm 0.13$ & $384(50)$ & $1.34 \pm 0.19$ & $359(48)$ & $1.01 \pm 0.18$ \\
\hline$\geq 1$ & $763(50)$ & $0.87 \pm 0.09$ & $386(50)$ & $0.95 \pm 0.13$ & $377(51)$ & $0.79 \pm 0.13$ \\
\hline 1-5 & $564(37)$ & $0.89 \pm 0.11$ & $270(35)$ & $0.94 \pm 0.15$ & $294(40)$ & $0.84 \pm 0.15$ \\
\hline $6-20$ & $153(10)$ & $0.86 \pm 0.18$ & $82(11)$ & $1.02 \pm 0.27$ & $71(10)$ & $0.68 \pm 0.22$ \\
\hline$>20$ & $46(3)$ & $0.77 \pm 0.38$ & $34(4)$ & $0.90 \pm 0.50$ & $12(2)$ & $0.36 \pm 0.23$ \\
\hline \multicolumn{7}{|c|}{ Clinically benign nevi } \\
\hline None & $59(4)$ & $1.33 \pm 0.55$ & $36(5)$ & $1.43 \pm 0.63$ & $23(3)$ & $1.17 \pm 1.09$ \\
\hline$\geq 1$ & 1444 (95) & $1.01 \pm 0.08$ & 731 (94) & $1.13 \pm 0.11$ & $713(96)$ & $0.88 \pm 0.11$ \\
\hline $1-5$ & $378(25)$ & $1.16 \pm 0.18$ & $205(26)$ & $1.35 \pm 0.25$ & $173(23)$ & $0.93 \pm 0.26$ \\
\hline $6-20$ & $649(43)$ & $1.06 \pm 0.12$ & $328(42)$ & $1.11 \pm 0.17$ & $321(43)$ & $1.01 \pm 0.17$ \\
\hline$>20$ & 417 (28) & $0.79 \pm 0.11$ & $198(26)$ & $0.94 \pm 0.19$ & $219(30)$ & $0.65 \pm 0.10$ \\
\hline \multicolumn{7}{|c|}{ Sunburn history } \\
\hline Yes & $1230(81)$ & $0.97 \pm 0.09$ & $627(81)$ & $1.08 \pm 0.12$ & $603(81)$ & $0.87 \pm 0.12$ \\
\hline No & $250(17)$ & $1.17 \pm 0.19$ & $128(17)$ & $1.34 \pm 0.30$ & $122(16)$ & $0.96 \pm 0.23$ \\
\hline
\end{tabular}

${ }^{\text {a }}$ Categories may not total $100 \%$ due to missing or unknown responses. Percentages of subgroups $1-5,6-20$, and $>20$ may not add to percentage of group $\geq 1$ due to rounding errors. $\pm 95 \%$ confidence interval.

II, or III $(1.00 \mathrm{~mm})$ was less than that in patients with skin types IV or V $(1.58 \mathrm{~mm})$, but this was not statistically significant $(P=0.1)$.

One half of the patients examined had at least one atypical nevus with similar percentages of males and females. The mean Breslow depth in both males and females with at least one atypical nevus $(0.95 \mathrm{~mm}$ and $0.79 \mathrm{~mm}$, respectively) was less than that in males and females without atypical nevi $(1.34 \mathrm{~mm}$ and $1.01 \mathrm{~mm}$, respectively; $P<0.001$ and $P<0.05$ ). Stratifying all patients with atypical nevi based on number 1-5, $6-20$, and greater than 20 revealed a decreasing trend in mean Breslow depths $(0.89 \mathrm{~mm}, 0.86 \mathrm{~mm}, 0.77 \mathrm{~mm}$, respectively), but there were no statistically significant differences between the groups. However, females with greater than 20 atypical nevi had significantly thinner lesions $(0.36 \mathrm{~mm})$ than females with no atypical nevi $(1.01 \mathrm{~mm}$; $P<0.001), 1-5(0.84 \mathrm{~mm} ; P$ $<0.001)$, and $6-20(0.68 \mathrm{~mm} ; P<0.05)$ atypical nevi.

The majority of patients had at least one clinically benign nevus. Only patients with greater than 20 clinically benign nevi had significantly thinner lesions $(0.79 \mathrm{~mm})$ than patients with $1-5(1.16 \mathrm{~mm} ; P<0.001)$ and $6-20(1.06 \mathrm{~mm} ; P=0.001)$ clinically benign nevi.

The majority of patients reported having at least one blistering or peeling sunburn with equal percentages of males and females. The mean Breslow depth of lesions in patients reporting a history of sunburn $(0.97$ $\mathrm{mm}$ ) was less than the mean depth in patients who recalled no history of sunburn $(1.17 \mathrm{~mm})$, but this was not statistically significant $(P=0.07)$.

\section{Detection Patterns}

The majority of lesions were detected by either the patient, physician, or spouse (Table 3). Physician-detected lesions were thinner $(0.40 \mathrm{~mm})$ than either self-detected $(1.17 \mathrm{~mm} ; P<0.001)$ or spouse-detected $(1.00 \mathrm{~mm} ; P<0.001)$ lesions.

In both genders, the majority of melanoma were self-detected. In males, the mean Breslow depth of self-detected lesions $(1.42 \mathrm{~mm})$ was greater than that of the lesions detected by either the spouse $(1.04 \mathrm{~mm}$; $P<0.005)$ or physician $(0.42 \mathrm{~mm} ; P<0.001)$. In females, the mean Breslow depth of self-detected lesions $(0.98 \mathrm{~mm})$ was greater than physician-detected lesions $(0.35 \mathrm{~mm} ; P<0.001)$ but was not significantly different from spouse-detected lesions $(0.72 \mathrm{~mm}$; $P$ $=0.2$ ).

Females $(76 \%)$ were more likely to self-detect their lesions than males $(52 \% ; P<0.001)$. Females selfdetected thinner melanomas $(0.98 \mathrm{~mm})$ than males (1.42 mm; $P<0.001)$. Males (19\%) were more likely than females $(3 \%)$ to have a lesion detected by their spouse $(P=0.001)$. Males $(19 \%)$ were also more likely than females $(9 \%)$ to have a lesion detected by their physician $(P=0.001)$. 
TABLE 3

Detection Patterns $^{\mathrm{a}}$

\begin{tabular}{|c|c|c|c|c|c|c|}
\hline \multirow[b]{2}{*}{ Detected by } & \multicolumn{2}{|c|}{ All ( $n=1630$ lesions) } & \multicolumn{2}{|c|}{ Males ( $n=849$ lesions) } & \multicolumn{2}{|c|}{ Females ( $n=781$ lesions) } \\
\hline & No. $(\%)$ & Mean depth (mm) & No. $(\%)$ & Mean depth (mm) & №. $(\%)$ & Mean depth (mm) \\
\hline Patient & $1,037(64)$ & $1.17 \pm 0.11$ & $445(52)$ & $1.42 \pm 0.18$ & $592(76)$ & $0.98 \pm 0.13$ \\
\hline Physician & $231(14)$ & $0.40 \pm 0.08$ & 159 (19) & $0.42 \pm 0.10$ & $72(9)$ & $0.35 \pm 0.14$ \\
\hline Spouse & $181(11)$ & $1.00 \pm 0.16$ & $161(19)$ & $1.04 \pm 0.17$ & $20(3)$ & $0.72 \pm 0.36$ \\
\hline Other ${ }^{\mathrm{b}}$ & $181(11)$ & & $84(10)$ & & $97(12)$ & \\
\hline
\end{tabular}

$\mathrm{a} \pm 95 \%$ confidence interval.

${ }^{\mathrm{b}}$ Other includes friend, sibling, son/daughter, parent, nurse, unknown/not available.

TABLE 4

Lesion Signs and Symptoms ${ }^{\mathbf{a}}$

\begin{tabular}{lll}
\hline Lesion sign/symptom & No. of lesions $(\boldsymbol{n}=\mathbf{1 6 3 0})(\%)$ & Mean depth $(\mathbf{m m})$ \\
\hline Color change & $703(43)$ & $1.15 \pm 0.12$ \\
Size change & $710(44)$ & $1.33 \pm 0.14$ \\
Shape/elevation change & $659(40)$ & $1.47 \pm 0.15$ \\
Ulceration & $149(9)$ & $2.69 \pm 0.37$ \\
Bleeding & $208(13)$ & $2.63 \pm 0.34$ \\
Tenderness & $151(9)$ & $2.44 \pm 0.42$ \\
Itching & $267(16)$ & $1.70 \pm 0.25$ \\
\hline & & \\
\hline
\end{tabular}

\section{Lesion Signs and Symptoms}

The most common changes noted by patients were the color, size, and/or shape/elevation of a lesion (Table 4). Less common changes included ulceration, bleeding, tenderness, and itching. Mean Breslow depths associated with a change in color $(1.15 \mathrm{~mm})$, size $(1.33 \mathrm{~mm})$, shape/elevation $(1.47 \mathrm{~mm})$, and itching $(1.70 \mathrm{~mm})$ were less than mean Breslow depths associated with ulceration $(2.69 \mathrm{~mm})$, bleeding $(2.63$ $\mathrm{mm})$, and tenderness $(2.44 \mathrm{~mm}$; all $P<0.005)$.

\section{Personal and Family History of Melanoma}

Of the 1630 cutaneous in situ and invasive melanomas, 1530 (94\%) were first primaries (15 lesions were concurrently diagnosed first primaries), 79 (5\%) were second primaries, 18 (1\%) were third primaries, and 3 were fourth or fifth primaries (Table 5). In both males and females, second $(0.46 \mathrm{~mm}$ and $0.34 \mathrm{~mm}$, respectively; $P<0.001$ and $P<0.001)$ and third $(0.31 \mathrm{~mm}$ and $0.24 \mathrm{~mm}$, respectively; $P<0.001$ and $P<0.005$ ) primary melanomas were detected earlier than first primary melanomas $(1.20 \mathrm{~mm}$ and $0.91 \mathrm{~mm}$, respectively). Although the mean Breslow depth of first primaries in females was less than that in males $(P$ $<0.001)$, the mean Breslow depths of second and third primaries were not significantly different between genders.
Fifteen percent of patients reported a blood relative with melanoma. The mean Breslow depth in patients with a family history of melanoma $(0.83 \mathrm{~mm})$ was less than that in patients without a family history of melanoma, but this was not statistically significant (1.02 mm; $P=0.08)$. The difference in Breslow depths between males with $(0.82 \mathrm{~mm})$ and without $(1.14 \mathrm{~mm})$ a family history of melanoma was statistically significant $(P<0.01)$.

\section{DISCUSSION}

Melanoma is a potentially curable cancer. Although the incidence and mortality rates have continued to increase, survival has also increased because of early diagnosis. ${ }^{4}$ Early detection of melanoma impacts morbidity and mortality rates and has substantial economic implications for the health care system. In 1997, the annual direct cost of treating newly diagnosed melanoma cases was about $\$ 563$ million. ${ }^{5}$ About $90 \%$ of the total cost was directed toward the treatment of patients with advanced disease. ${ }^{5}$ Public education campaigns may increase awareness and knowledge of the disease, which may lead to behavior changes that have an impact on both primary prevention and early detection. ${ }^{6}$ Similarly, professional education of physicians and integration of skin examinations into physical examinations may lead to early detection. ${ }^{6}$ Early detection of melanoma may have a relatively immediate impact on decreasing mortality rates. ${ }^{7}$ In the U.S. population, deficiencies in knowledge and practices promoting early detection have shown the need for public education. ${ }^{8}$ Miller et al. ${ }^{8}$ surveyed a nationally representative sample of 1001 persons in the United States. They found that nearly $42 \%$ were unaware of the term melanoma and only $26 \%$ of those who were aware could identify its specific signs. Awareness and knowledge were more common among women, Caucasians, adults over 25 years of age, and among those with higher levels of education and income. They also discovered that $54 \%$ of 
TABLE 5

Personal and Family History of Melanoma ${ }^{a}$

\begin{tabular}{|c|c|c|c|c|c|c|}
\hline & \multicolumn{2}{|c|}{ All } & \multicolumn{2}{|c|}{ Males } & \multicolumn{2}{|c|}{ Females } \\
\hline & No. $(\%)$ & Mean depth (mm) & No. $(\%)$ & Mean depth (mm) & No. $(\%)$ & Mean depth (mm) \\
\hline \multicolumn{7}{|l|}{ Personal history ${ }^{\mathrm{b}}$} \\
\hline Primary & \multicolumn{2}{|c|}{$n=1630$ lesions } & \multicolumn{2}{|c|}{$n=849$ lesions } & \multicolumn{2}{|c|}{$n=781$ lesions } \\
\hline 1st & 1530 (94) & $1.06 \pm 0.08$ & 784 (92) & $1.20 \pm 0.12$ & 746 (96) & $0.91 \pm 0.11$ \\
\hline 2nd & $79(5)$ & $0.42 \pm 0.16$ & $54(6)$ & $0.46 \pm 0.22$ & $25(3)$ & $0.34 \pm 0.16$ \\
\hline $3 r d$ & $18(1)$ & $0.28 \pm 0.17$ & $10(1)$ & $0.31 \pm 0.21$ & $8(1)$ & $0.24 \pm 0.35$ \\
\hline 4th & 2 & 0.44 & 1 & 0.46 & 1 & 0.41 \\
\hline 5th & 1 & 3.83 & 0 & & 1 & 3.83 \\
\hline Family history ${ }^{\mathrm{c}}$ & \multicolumn{2}{|c|}{$n=1515$ patients } & \multicolumn{2}{|c|}{$n=774$ patients } & \multicolumn{2}{|c|}{$n=741$ patients } \\
\hline Yes & 231 (15) & $0.83 \pm 0.20$ & 103 (13) & $0.82 \pm 0.19$ & $128(17)$ & $0.83 \pm 0.33$ \\
\hline No & $1257(83)$ & $1.02 \pm 0.08$ & $654(84)$ & $1.14 \pm 0.12$ & $603(81)$ & $0.89 \pm 0.11$ \\
\hline
\end{tabular}

respondents never examined their skin because of low awareness, little knowledge, or denial of risk. These authors concluded that future educational programs should focus on increasing concern and knowledge of melanoma. ${ }^{8}$ Oliveria et al. ${ }^{9}$ showed that awareness of skin changes was correlated with a thinner melanoma and more immediate medical attention and that knowledge of the signs and symptoms also contributed to less delay in diagnosis. Campaigns to increase awareness and promote skin self-examinations have been developed by the American Academy of Dermatology. To gain further insight into targeting public and professional educational campaigns for early detection, this study evaluated the relationship between tumor thickness and patient demographics, detection patterns, lesion signs and symptoms, and personal and family history of melanoma. This is the largest prospective study to evaluate the relationship between Breslow depth and detection patterns, lesion characteristics, and patient characteristics.

\section{Patient Demographics}

Educating and screening patients at high risk for melanoma (skin types I, II, or III, atypical nevi, many clinically benign nevi, significant sun exposure, or personal or family history of melanoma) about skin selfexamination and physician examinations will continue to promote the early detection of thin melanoma. ${ }^{10-12}$ However, strategies aimed at educating only this population will fail to make an impact on decreasing the incidence of deeper melanomas that were seen in low-risk groups in this study. Although high-risk groups are more likely, by definition, to have melanoma, our data indicate that this group already tends to present with early disease. Based on our data, we suggest that education to promote early detection should specifically address low-risk groups because thicker lesions are found more frequently among these individuals.

In our study, patients with skin types I, II, or III had thinner lesions than patients with skin types IV or $\mathrm{V}$. Although not statistically significant, this is consistent with our clinical impression and the published literature. ${ }^{13}$ This may suggest less awareness and knowledge of melanoma in non-Caucasians. Miller et $\mathrm{al}^{8}$ found lower measures of melanoma knowledge and attitudes among non-Caucasians compared with Caucasians.

Our study showed that patients with atypical nevi had thinner melanomas than patients without atypical nevi. This earlier detection may be a result of increased screening and surveillance of these patients because of awareness of a higher risk of melanoma. ${ }^{10}$ In our study, patients with greater than 20 clinically benign nevi had significantly thinner melanomas than patients with 20 or less clinically benign nevi.

The patients in this study who reported a history of sunburn had thinner lesions than patients who recalled no history of sunburn, although this was not statistically significant. An awareness of sunburn as a risk factor for melanoma ${ }^{14}$ and other skin carcinomas by either patient or physician may lead to increased surveillance by both patients and physicians and to detection of earlier disease in patients who give a history of sunburn independent of actual exposure. 


\section{Detection Patterns}

Educational campaigns for the public are essential because the majority of lesions are not detected by health care professionals. Our study and studies by Koh et al., ${ }^{15}$ Brady et al., ${ }^{16}$ and Epstein et al. ${ }^{17}$ showed that the majority of melanomas were detected by patients. Similar percentages were noted among the four studies, i.e., $64 \%, 53 \%, 57 \%$, and $55 \%$, respectively. Our study and others also showed that females were more likely than males to detect their own lesions ${ }^{15-17}$ and this study showed that the lesions detected were thinner. This may be accounted for by the findings of other investigators that women are more aware of the disease, more likely to participate in screening programs and perform skin self-examinations, and more likely to seek early medical evaluation of lesions. ${ }^{8,18}$ Therefore, educational programs should specifically include increasing melanoma awareness among males.

Physicians who detect melanomas tend to identify early lesions. Our study showed that physician-detected lesions were significantly thinner than either self-detected or spouse-detected lesions. Brady et al. ${ }^{16}$ and Epstein et al. ${ }^{17}$ also concluded that thinner melanomas were more likely to be physician detected. However, physician detection was less common than patient detection among the three studies. One possible explanation is that few physicians examine the skin of their patients. Geller et al. ${ }^{19}$ reported that $87 \%$ of 216 melanoma patients had regular physicians and $63 \%$ saw their physicians in the year before diagnosis. Many patients did not have skin examinations by their physicians nor did they perform skin self-examinations. Few physicians have little or no training in the identification of melanomas, particularly the early detection of these lesions. Stephenson et al. ${ }^{20}$ found that greater than $50 \%$ of family physicians lacked confidence in recognizing melanoma. Cassileth et al. ${ }^{21}$ showed that only $38 \%$ of nondermatologist physicians correctly identified at least four of six melanomas compared with $92 \%$ of dermatologists. Perhaps, more comprehensive training of physicians, particularly primary care physicians, at identifying early melanomas and encouraging them to perform skin examinations as part of health maintenance may increase the detection of early disease.

\section{Lesion Signs and Symptoms}

Both the public and physicians should be educated as to the early signs of melanoma such as a change in color, size, or shape, to maximize the detection of early disease. Our study showed that thinner lesions were associated with a change in color, size, shape/ elevation, or itching, whereas ulceration, bleeding, or tenderness were signs of significantly thicker lesions. This is compatible with the findings of other investigators. Wick et al. ${ }^{22}$ and Sober et al. ${ }^{23}$ showed that a change in color and an increase in size were most useful in the detection of early lesions, whereas elevation, bleeding, or ulceration were associated more frequently with thicker melanomas. Based on the results of all these studies, it is reasonable to conclude that educational campaigns promoting the early signs and symptoms of melanoma, including a change in color, size, or shape, or pruritus, may increase the detection of early disease.

\section{Personal and Family History of Melanoma}

A previous personal history of melanoma is a strong risk factor for the development of subsequent melanomas,$^{24}$ frequently leading to regular physician and skin self-examinations. Several investigators studying patients with multiple primary melanomas have reported a decrease in thickness from first to subsequent primaries. ${ }^{11,12}$ In our study, second and third primary melanomas were detected earlier than first primary melanomas. First primaries in females were significantly thinner than in males. However, Breslow depths of second and third primaries were comparable in both males and females, suggesting that a personal history of melanoma impacts positively on education of and surveillance in males.

Similarly, patients with a family history of melanoma may already have an increased awareness of melanoma. Brady et al. ${ }^{16}$ found that patients who reported a family history of melanoma were more likely to present with a thin lesion. Our study also concluded that patients with a family history of melanoma had earlier detection of disease than patients without a family history of melanoma, although this was not statistically significant. However, there was a significant difference in the thickness of lesions in males with and without a family history of melanoma. This observation suggests that not only a personal but also a family history of melanoma impacts positively on education of and surveillance in males.

In summary, patient characteristics associated with early detection included female gender, at least one atypical nevus, greater than 20 clinically benign nevi, and/or a personal history of melanoma. Skin types I, II, and III, history of sunburn, and/or a family history of melanoma were also associated with thinner lesions, but not at a statistically significant level. Lesion characteristics associated with earlier detection included a change in color, size, shape/elevation, and/or itching. Physician-detected melanomas were significantly thinner but represented a relatively small 
percentage of melanomas. This study suggests that educational campaigns that increase melanoma awareness and screening in males may be beneficial. Although screening only high-risk groups may be economically advantageous, our data suggest that educational campaigns to increase melanoma awareness should be directed at both high and low-risk groups. Physicians and other health care providers should be aware of the importance and potential benefit of total body skin examinations for both high and low-risk patients.

\section{REFERENCES}

1. Wingo PA, Ries LAG, Rosenberg HM, Miller DS, Edwards BK. Cancer incidence and mortality, 1973-1995: a report card for the U.S. Cancer. 1998;82:1197-1207.

2. Rigel DS, Friedman RJ, Kopf AW. The incidence of malignant melanoma in the United States: issues as we approach the 21st century. J Am Acad Dermatol. 1996;34:839-847.

3. Greenlee RT, Murray T, Bolden S, Wingo PA. Cancer statistics, 2000. CA. 2000;50:7-33.

4. Sober AJ. Cutaneous melanoma: opportunity for cure. CA. 1991;41:197-199.

5. Tsao H, Rogers GS, Sober AJ. An estimate of the annual direct cost of treating cutaneous melanoma. J Am Acad Dermatol. 1998;38:669-680.

6. Koh HK, Geller AC, Miller DR, Lew RA. The current status of melanoma early detection and screening. Dermatol Clin. 1995;13:623-634.

7. Marks R. Prevention and control of melanoma: the public health approach. CA. 1996;46:199-216.

8. Miller DR, Geller AC, Wyatt SW, et al. Melanoma awareness and self-examination practices: results of a United States survey. J Am Acad Dermatol. 1996;34:962-970.

9. Oliveria SA, Christos PJ, Halpern AC, Fine JA, Barnhill RL, Berwick M. Patient knowledge, awareness, and delay in seeking medical attention for malignant melanoma. J Clin Epidemiol. 1999;52:1111-1116.

10. Masri GD, Clark WH, Guerry DI, Halpern A, Thompson CJ, Elder DE. Screening and surveillance of patients at high risk for malignant melanoma result in detection of earlier disease. J Am Acad Dermatol. 1990;22:1042-1048.

11. Kang S, Barnhill RL, Mihm MC, Sober AJ. Multiple primary cutaneous melanomas. Cancer. 1992;70:1911-1916.

12. Johnson TM, Hamilton TA, Lowe L. Multiple primary melanomas. J Am Acad Dermatol. 1998;39:422-427.

13. Reintgen DS, McCarty KM, Cox E, Seigler HF. Malignant melanoma in black American and white American populations: a comparative review. J Am Med Assoc. 1982;248:18561859.

14. Elwood JM, Jopson J. Melanoma and sun exposure: an overview of published studies. Int J Cancer. 1997;73:198-203.

15. Koh HK, Miller DR, Geller AC, Clapp RW, Mercer MB, Lew RA. Who discovers melanoma? Patterns from a populationbased survey. J Am Acad Dermatol. 1992;26:914-919.

16. Brady MS, Oliveria SA, Christos PJ, et al. Patterns of detection in patients with cutaneous melanoma. Cancer. 2000;89: 342-347.

17. Epstein DS, Lange JR, Gruber SB, Mofid M, Koch SE. Is physician detection associated with thinner melanomas? J Am Med Assoc. 1999;281:640-643.

18. Robinson JK, Rigel DS, Amonette RA. What promotes skin self-examination? J Am Acad Dermatol. 1998;38:752-757.

19. Geller AC, Koh HK, Miller DR, Clapp RW, Mercer MB, Lew RA. Use of health services before the diagnosis of melanoma: implications for early detection and screening. J Gen Intern Med. 1992;7:154-157.

20. Stephenson A, From L, Cohen A, Tipping J. Family physicians' knowledge of malignant melanoma. J Am Acad Dermatol. 1997;37:953-957.

21. Cassileth BR, Clark WH, Lusk EJ, Frederick BE, Thompson CJ, Walsh WP. How well do physicians recognize melanoma and other problem lesions? J Am Acad Dermatol. 1986;14: $555-560$.

22. Wick MM, Sober AJ, Fitzpatrick TB, et al. Clinical characteristics of early cutaneous melanoma. Cancer. 1980;45:26842686.

23. Sober AJ, Day CL, Kopf AW, Fitzpatrick TB. Detection of thin primary melanomas. CA. 1983;33:160-163.

24. Tucker MA, Boice JD, Hoffman DA. Second cancer following cutaneous melanoma and cancers of the brain, thyroid, connective tissue, bone, and eye in Connecticut, 1935-82. Natl Cancer Inst Monogr. 1985;68:161-189. 